\title{
An elementary bound on Siegel zeroes
}

\author{
Thomas Morrill* \\ School of Physical, Environmental and Mathematical Sciences \\ The University of New South Wales Canberra, Australia \\ t.morrill@adfa.edu.au \\ Tim Trudgian* ${ }^{\dagger}$ \\ School of Physical, Environmental and Mathematical Sciences \\ The University of New South Wales Canberra, Australia \\ t.trudgian@adfa.edu.au
}

December 3, 2018

\begin{abstract}
We consider Dirichlet $L$-functions $L(s, \chi)$ where $\chi$ is a real, non-principal character modulo $q$. Using Pintz's refinement of Page's theorem, we prove that for $q \geq 3$ the function $L(s, \chi)$ has at most one real zero $\beta$ with $1-1.011 / \log q<\beta<1$.
\end{abstract}

\section{Introduction}

Let $\chi(n)$ be a real, non-principal Dirichlet character to the modulus $q$ and let $L(s, \chi)$ be the associated Dirichlet $L$-function, where $s=\sigma+i t$. It is known [2, pp. 93-95] that $L(s, \chi)$ has at most one zero with real part larger than $1-(A \log \max \{q, q|t|\})^{-1}$. Such an exceptional, or Siegel, zero must lie on the real axis. A classical result of Page [9] is that, given a single character $\chi$ modulo $q$, there can be at most one exceptional zero 'close' to unity.

Theorem 1 (Page). If $\chi$ mod $q$ is a real, non-principal character, and if $\beta_{1}$ and $\beta_{2}$ are real zeroes of $L(s, \chi)$, then there is a positive constant $c_{1}$ such that

$$
\min \left(\beta_{1}, \beta_{2}\right) \leq 1-\frac{c_{1}}{\log q} .
$$

\footnotetext{
*Supported by Australian Research Council Discovery Project DP160100932

${ }^{\dagger}$ Supported by Australian Research Council Future Fellowship FT160100094.
} 
McCurley [6] and Kadiri [4 have given values for $c_{1}$ : the best is $c=0.909$ by Kadiri. Kadiri's method, similar to that in papers on the zero-free region of $L$-functions (see, e.g. [5] and [7]), uses a special nonnegative trigonometric polynomial, the calculus of variations, and an analysis of the distribution of the imaginary parts of zeroes of $L(s, \chi)$.

Pintz [10, Thm 2] revisits Page's method, which is more elementary. Using the PólyaVinogradov inequality, Pintz is able to prove that $c_{1}=2+o(1)$ as $q \rightarrow \infty$. Indeed, he notes that one can use the Burgess bounds on character sums to improve this to $c_{1}=4+o(1)$. In making these results explicit there will be some loss in the size of $c_{1}$. We aim to minimise this loss by using the best off-the-shelf explicit estimates. Our main result is the following.

Theorem 2. If $\chi \bmod q$ is a real, non-principal character, with $q \geq 3$, and if $\beta_{1}$ and $\beta_{2}$ are real zeroes of $L(s, \chi)$, then

$$
\min \left(\beta_{1}, \beta_{2}\right) \leq 1-\frac{1.011}{\log q} .
$$

Throughout the course of the paper we take take $\chi$ to be a primitive character. This is no great obstacle, since, as noted by Pintz [10, p. 164], if $\chi$ modulo $q$ is induced by a primitive character $\chi^{\prime}$ modulo $q^{\prime}$, then if $L(\beta, \chi)=0$ we have $L\left(\beta, \chi^{\prime}\right)=0$. Since $q \leq q^{\prime}$, we can therefore extend the result to that in Theorem 2 ,

The rest of this paper is organised as follows. In $\$ 2$ we collect some preliminary results. We use these, in $\$ 3$ to refine Pintz's result for finite ranges of $q$. We detail, in \$3.1, our computations. These prove Theorem 2 for finite ranges of $q$; we then use Pintz's original argument for large $q$. Finally, in $\$ 4$ we outline some potential improvements to our results.

Throughout the paper $\vartheta$ will denote a complex number with modulus at most unity.

\section{Preliminary lemmas}

In this section we collect some results from the literature. We first note that we need not concern ourselves with small values of $q$. Watkins [14] showed that there are no Siegel zeroes for $L(s, \chi)$ where $\chi$ is odd, and $q \leq 3 \cdot 10^{8}$; Platt [11] reached the same conclusion for $\chi$ even and $q \leq 4 \cdot 10^{5}$.

We wish to record an explicit version of the Pólya-Vinogradov inequality.

Lemma 1 (Frolenkov and Soundararajan [3]). Let $\chi$ be a primitive character with parity $\chi(-1)=(-1)^{i}$. We have for all $q \geq 1200$, that

$$
\left|\sum_{n=M+1}^{M+N} \chi(n)\right| \leq E_{i} \sqrt{q} \log q+\sqrt{q},
$$

where $E_{0}=2 / \pi^{2}, E_{1}=1 / 2 \pi$.

We note that, by a remark of Pomerance [12], we can divide the right-side of (1) by two if $M=0$ and $\chi$ is even. Define

$$
A\left(q_{0}\right)=A_{i}\left(q_{0}\right):= \begin{cases}1 / \pi^{2}+1 /\left(2 \log q_{0}\right), & \text { if } i=0 \\ 1 / 2 \pi+1 / \log q_{0}, & \text { if } i=1,\end{cases}
$$


so that, for $q \geq q_{0} \geq 1200$,

$$
\left|\sum_{n=M+1}^{M+N} \chi(n)\right| \leq A\left(q_{0}\right) \sqrt{q} \log q .
$$

We shall make use of the Euler-Maclaurin summation formula — see [8, Thm 2.19].

Lemma 2 (Euler-Maclaurin summation). Let $k$ be a nonnegative integer and $f(x)$ be $(k+1)$ times differentiable on the interval $[a, b]$. Then

$$
\begin{aligned}
\sum_{a<n \leq b} f(n) & =\int_{a}^{b} f(t) d t+\sum_{r=0}^{k} \frac{(-1)^{r+1}}{(r+1) !}\left(f^{(r)}(b)-f^{(r)}(a)\right) B_{r+1} \\
& +\frac{(-1)^{k}}{(k+1) !} \int_{a}^{b} B_{k+1}(x) f^{(k+1)}(x) d x,
\end{aligned}
$$

where $B_{j}(x)$ is the $j$ th periodic Bernoulli polynomial and $B_{j}=B_{j}(0)$.

Pintz takes $k=0$ and examines sums of $n^{-\alpha}$ and $(\log n) n^{-\alpha}$. We shall require more precision for our calculations. Choosing $k=2$ we have, for $0<\alpha<1$,

$$
\sum_{n \leq x} n^{-\alpha}=C_{1}(\alpha)+\frac{1}{1-\alpha}\left(x^{1-\alpha}-1\right)+\frac{1}{2} x^{-\alpha}-\frac{\alpha}{12} x^{-\alpha-1}+\vartheta \frac{\alpha(\alpha+1)}{72 \sqrt{3}} x^{-\alpha-2},
$$

where

$$
C_{1}(\alpha)=\frac{1}{2}+\frac{\alpha}{12}+\frac{\alpha(\alpha+1)(\alpha+2)}{6} \int_{1}^{\infty} \frac{\{t\}^{3}-\frac{3}{2}\{t\}^{2}+\frac{1}{2}\{t\}}{t^{\alpha+3}} d t .
$$

Similarly, with $k=2$ we have for $0<\alpha<1$

$$
\begin{aligned}
\sum_{n \leq x}(\log n) n^{-\alpha}= & C_{2}(\alpha)+\frac{x^{1-\alpha} \log x}{1-\alpha}+\frac{1}{(1-\alpha)^{2}}\left(1-x^{1-\alpha}\right)+\frac{1}{2} x^{-\alpha} \log x \\
& +\vartheta x^{-\alpha-1}\left\{\alpha \log x\left(1+\frac{1}{72 \sqrt{3}}\right)+1+\frac{3 \alpha+1}{72 \sqrt{3}(\alpha+1)}\right\},
\end{aligned}
$$

where

$$
C_{2}(\alpha)=1-\frac{1}{12}+\frac{1}{6} \int_{1}^{\infty} \frac{(\alpha(\alpha+1) \log t-1-2 \alpha)\left(\{t\}^{3}-\frac{3}{2}\{t\}^{2}+\frac{1}{2}\{t\}\right)}{t^{\alpha+2}} d t
$$

The class-number formula allows one to show [2, p. 95] that $L(1, \chi) \gg q^{-1 / 2}$. We require an explicit version of this as given by Bennett, Martin, O'Bryant and Rechnitzer [1, Lem. 6.3 and A.9].

Lemma 3 (Bennett et al.). If $\chi$ modulo $q$ is a real primitive character, then

$$
L(1, \chi) \geq \begin{cases}79.2 q^{-1 / 2}, & \text { if } 4 \cdot 10^{5} \leq q \leq 10^{7} \\ 12 q^{-1 / 2}, & \text { if } q>10^{7}\end{cases}
$$


Consider

$$
\sum_{n \leq x} \frac{g(n) \log n}{n^{1-\tau}}, \quad g(n)=\sum_{d \mid n} \chi(d)
$$

where $0<\tau<1$. As in Pintz, we note that

$$
g(n)=\prod_{p^{e} \| n}\left(1+\chi(p)+\chi\left(p^{2}\right)+\cdots+\chi\left(p^{e}\right)\right)
$$

Since $\chi(n)$ is absolutely multiplicative we have that $g\left(m^{2}\right) \geq 1$. Pintz uses this to show that the first sum in (44) exceeds $(\log 4) / 4$ for $x \geq 4$. We improve this, using partial summation.

\section{Lemma 4.}

$$
\sum_{n \leq x} \frac{g(n) \log n}{n^{1-\tau}} \geq-2 \zeta^{\prime}(2-2 \tau)-f(2 \tau-1, x)
$$

where

$$
f(\alpha, x)=\frac{2\left(x^{1 / 2}-1\right)^{\alpha}\left(1-\alpha \log \left(x^{1 / 2}-1\right)\right)}{\alpha^{2}}, \quad\left(\log \left(x^{1 / 2}-1\right)>\frac{1}{2 \tau-1}\right) .
$$

We note that we shall only apply (5) for finite values of $x$, and, as such, we can avoid the usual irritation about bounding terms such as $x^{1 / 2}-1$ from below.

Finally, by partial summation we have, for any continuously differentiable function $h(z)$,

$$
\sum_{x<n \leq y} \chi(n) h(n)=h(y) \sum_{1 \leq n \leq y} \chi(n)-h(x) \sum_{1 \leq n \leq x} \chi(n)-\int_{x}^{y} h^{\prime}(t) \sum_{1 \leq n \leq t} \chi(n) d t .
$$

Suppose that $h$ is a positive decreasing function with $\lim _{z \rightarrow \infty} h(z)=0$. For our purposes, $h \in\left\{(\log z) / z^{1-\tau}, 1 / z^{1-\tau}, 1 / z\right\}$. For any $x$ we have $\sum_{n \leq x} \chi(n) \ll \sqrt{q} \log q$, by the PólyaVinogradov inequality, whence

$$
\left|\sum_{n>x} \chi(n) h(n)\right| \leq h(x)\left|\sum_{1 \leq n \leq x} \chi(n)\right|+\int_{x}^{\infty}\left|h^{\prime}(t)\right|\left|\sum_{1 \leq n \leq t} \chi(n)\right| d t .
$$

Depending on the size of $z$ we shall use a mixture of the trivial bound and the PólyaVinodgradov inequality in (6). Doing this, and integrating by parts, yields

$$
\left|\sum_{n>z} \chi(n) h(n)\right| \leq \begin{cases}2 z h(z)+\int_{z}^{A\left(q_{0}\right) \sqrt{q} \log q} h(t) d t, & \text { if } z \leq A\left(q_{0}\right) \sqrt{q} \log q \\ 2 A\left(q_{0}\right) \sqrt{q}(\log q) h(z), & \text { if } z \geq A\left(q_{0}\right) \sqrt{q} \log q,\end{cases}
$$

where $A\left(q_{0}\right)$ is defined in (2) . 


\section{Outline of proof}

Following Pintz, we apply our Lemma 2 to obtain

$$
\sum_{n \leq x} \frac{g(n) \log n}{n^{1-\tau}}=T_{1}+T_{2}-T_{3}-\frac{(1-\tau)}{12} x^{\tau-2} \sum_{d \leq x} \chi(d) d \log d+\frac{1}{2} x^{\tau-1} \log x \sum_{d \leq x} \chi(d)+\vartheta W
$$

where

$$
\begin{aligned}
& T_{1}=K_{1} \sum_{d \leq x} \chi(d) \frac{\log d}{d^{1-\tau}} \\
& T_{2}=K_{2} \sum_{d \leq x} \chi(d) \frac{1}{d^{1-\tau}} \\
& T_{3}=\frac{x}{\tau}\left(\frac{1}{\tau}-\log x\right) \sum_{d \leq x} \chi(d) \frac{1}{d} .
\end{aligned}
$$

Here, $K_{1}$ and $K_{2}$ are positive constants depending only on $\tau$, and

$$
\begin{aligned}
W= & x^{\tau} \log x \frac{(1-\tau)(2-\tau)}{432 \sqrt{3}}\left(2+x_{0}^{-1}\right)\left(1+x_{0}^{-1}\right) \\
& +\frac{x^{\tau}}{2}\left(1+x_{0}\right)^{-1}\left((1-\tau)\left(1+\frac{1}{72 \sqrt{3}}\right) \log x+1+\frac{4-3 \tau}{72 \sqrt{3}(2-\tau)}\right) .
\end{aligned}
$$

Note that Pintz introduces the variable $z \leq x$ and splits the left-side of (8) to bound what would otherwise be $x^{1+\tau} \log x$ in (3.5) of [10]. This is rendered unnecessary by using (3).

We bound the character sums in (8) by using (7). This produces

$$
\sum_{n \leq x} \frac{g(n) \log n}{n^{1-\tau}}=K_{1} L^{\prime}(1-\tau)+K_{2} L(1-\tau)-\frac{x}{\tau}\left(\frac{1}{\tau}-\log x\right) L(1)+E(q, \tau, x),
$$

where $E$ is an unwieldy, though easily computed, error term. Assume, that there are two zeroes of $L(s, \chi)$ with $s$ in the interval $(1-c / \log q, 1)$. Hence there is a value of $\tau \in(0, c / \log q)$ for which $L(1-\tau) \leq 0$ and $L^{\prime}(1-\tau)=0$. We therefore have

$$
\sum_{n \leq x} \frac{g(n) \log n}{n^{1-\tau}} \leq E(q, \tau, x)-\left(\frac{1}{\tau}-\log x\right) \frac{B x^{\tau}}{\tau \sqrt{q}}, \quad\left(\tau^{-1} \geq \log x\right),
$$

where $B$ is either 79.2 or 12 , according to Lemma 3. We now invoke Lemma 4, which provides us with a contradiction if $F=F(q, \tau, x)<0$, where

$$
F=E(q, \tau, x)-\left(\frac{1}{\tau}-\log x\right) \frac{B x^{\tau}}{\tau \sqrt{q}}+2 \zeta^{\prime}(2-2 \tau)+2\left(\frac{1+\left(\frac{1}{2}-\tau\right) \log x_{0}}{x_{0}^{1 / 2-\tau}(1-2 \tau)^{2}}\right)<0
$$

subject to $x_{0} \leq x \leq \exp \left(\tau^{-1}\right)$. 


\begin{tabular}{ll|ll|ll}
\hline \hline$q_{0}$ & $q_{1}$ & $c_{\text {even }}$ & $x_{\text {even }}$ & $c_{\text {odd }}$ & $x_{\text {odd }}$ \\
\hline $4 \cdot 10^{5}$ & $7 \cdot 10^{5}$ & 1.011 & $10^{5.54}$ & - & - \\
$7 \cdot 10^{5}$ & $10^{6}$ & 1.017 & $10^{5.73}$ & - & - \\
$10^{6}$ & $1.7 \cdot 10^{6}$ & 1.020 & $10^{5.88}$ & - & - \\
$1.7 \cdot 10^{6}$ & $3.1 \cdot 10^{6}$ & 1.025 & $10^{6.08}$ & - & - \\
$3.1 \cdot 10^{6}$ & $6.1 \cdot 10^{6}$ & 1.030 & $10^{6.30}$ & - & - \\
$6.1 \cdot 10^{6}$ & $1.3 \cdot 10^{7}$ & 1.036 & $10^{6.54}$ & - & - \\
$1.3 \cdot 10^{7}$ & $2.4 \cdot 10^{7}$ & 1.041 & $10^{6.83}$ & - & - \\
$2.4 \cdot 10^{7}$ & $5.4 \cdot 10^{7}$ & 1.044 & $10^{7.06}$ & - & - \\
$5.4 \cdot 10^{7}$ & $1.5 \cdot 10^{8}$ & 1.051 & $10^{7.35}$ & - & - \\
$1.5 \cdot 10^{8}$ & $6.2 \cdot 10^{8}$ & 1.055 & $10^{7.75}$ & 1.021 & $10^{8.00}$ \\
$6.2 \cdot 10^{8}$ & $4.4 \cdot 10^{9}$ & 1.060 & $10^{8.29}$ & 1.029 & $10^{8.54}$ \\
$4.4 \cdot 10^{9}$ & $6.4 \cdot 10^{10}$ & 1.070 & $10^{9.01}$ & 1.041 & $10^{9.26}$ \\
$6.4 \cdot 10^{10}$ & $2.7 \cdot 10^{12}$ & 1.080 & $10^{10.00}$ & 1.055 & $10^{10.24}$ \\
$2.7 \cdot 10^{12}$ & $6.2 \cdot 10^{14}$ & 1.090 & $10^{11.40}$ & 1.069 & $10^{11.63}$ \\
$6.2 \cdot 10^{14}$ & $2.1 \cdot 10^{18}$ & 1.101 & $10^{13.43}$ & 1.082 & $10^{13.66}$ \\
$2.1 \cdot 10^{18}$ & $4.4 \cdot 10^{21}$ & 1.200 & $10^{15.26}$ & 1.182 & $10^{15.50}$ \\
$4.4 \cdot 10^{21}$ & $1.5 \cdot 10^{24}$ & 1.300 & $10^{16.64}$ & 1.283 & $10^{16.86}$ \\
$1.5 \cdot 10^{24}$ & $3.1 \cdot 10^{26}$ & 1.350 & $10^{17.90}$ & 1.334 & $10^{18.12}$ \\
$3.1 \cdot 10^{26}$ & $2.4 \cdot 10^{28}$ & 1.400 & $10^{18.92}$ & 1.383 & $10^{19.15}$ \\
$2.4 \cdot 10^{28}$ & $1.5 \cdot 10^{30}$ & 1.425 & $10^{19.91}$ & 1.411 & $10^{20.11}$ \\
$1.5 \cdot 10^{30}$ & $10^{32}$ & 1.445 & $10^{20.89}$ & 1.429 & $10^{21.11}$ \\
$10^{32}$ & $9.1 \cdot 10^{32}$ & 1.495 & $10^{21.40}$ & 1.480 & $10^{21.61}$ \\
\hline \hline
\end{tabular}

Table 1: Values of $c$ and $x$ so that $F(q, \tau, x)<0$ for $q \in\left[q_{0}, q_{1}\right]$.

\subsection{Algorithm}

We wish to calculate the best constant $c$ in Theorem 2 on some range $q_{0} \leq q \leq q_{1}$. We calculate an upper bound $F^{\star}$ so that $F \leq F^{\star}$ for all $q \in\left[q_{0}, q_{1}\right]$. For a fixed $c$, it suffices to find $0<x^{\star} \leq \exp \left(\tau^{-1}\right)$ so that $F^{\star}(q, c / \log q, x)<0$. The algorithm calculates $F^{\star}$ at test points $x^{\star}$; if an $x^{\star}$ is found so that $F^{\star}<0$, the algorithm increments $c$ and restarts the search. When no admissible $x^{\star}$ values are found, the algorithm terminates and returns the last known $c$ and $x^{\star}$ values for which Theorem 2 is true.

The algorithm is run separately for even and odd characters, using the Pólya-Vinogradov bounds in Lemma 1. The results of this computation are given in Table1, This computation was run in Sage on a $2.9 \mathrm{GHz}$ processor. The code is available at https://github.com/tsmorrill/Pintz.

\subsection{Large moduli}

For $q$ outside Table 1, we apply Lemma 3 of [10] with $x=A \sqrt{q}(\log q) / \tau^{8}$, where $A=A\left(q_{0}\right)$ as defined in (2). Suppose $L(s, \chi)$ has two zeroes in the interval $(1-c / \log q, 1)$. Pintz 
considers a variation of (9): to obtain a contradiction he requires

$$
\frac{6 e c}{\log q}<\frac{\log 4}{4} \quad \text { and } \quad \frac{1}{\tau}-\log x>0 .
$$

For $c=1.011$, a quick check shows that we need $q \geq 4.6 \cdot 10^{20}$ for the first inequality in (10) to hold. Consider the second inequality: for $\tau \leq c / \log q$, we have that

$$
1 / \tau-\log x=1 / \tau-\log A-\log (\sqrt{q} \log q)-8 \log 1 / \tau
$$

If we have $\tau<1 / 8$, then (11) is decreasing - fortunately, this is implied by $6 e c / \log q<$ $(\log 4) / 4$. Therefore, we have

$$
1 / \tau-\log x \geq\left(\frac{1}{c}-\frac{1}{2}\right) \log q-\log A-9 \log \log q+8 \log c
$$

for $q \geq 4.6 \cdot 10^{20}$. To ensure that the right-side of (12) is positive, we need $q>2.6 \cdot 10^{32}$ for even characters, and $q>9.1 \cdot 10^{32}$ for odd characters. Thus, Theorem 2 holds for all $q>9.1 \cdot 10^{32}$. This, along with Table 1 completes the proof.

We note that the argument leading to the contradiction could be improved by replacing the $(\log 4) / 4$ bound with the result from Lemma 4. However, the more difficult inequality to satisfy is (12), and retaining the $(\log 4) / 4$ eases the computation.

\section{Conclusions}

Our result can be improved at several places. A marginally better constant in the PólyaVinogradov inequality in Lemma 1 gives little overall improvement. Similarly, if one extended the computation done by Bennett et al. [1], and dealt with some small values of $q$ directly, one may improve slightly on the lower bounds on $L(1, \chi)$ in Lemma 3 .

More importantly, the small values of $q$ we are forced to consider impede our calculation of $c$. If Platt's result were extended to show that there are no Siegel zeroes for $L(s, \chi)$ for $\chi$ even and $q \leq Q$ where $Q>4 \cdot 10^{5}$, then Theorem 2 may be improved according to Table 1 . Note that odd characters above $3 \cdot 10^{8}$ must also be dealt with to improve $c \geq 1.02$.

We have essentially 'lost half' of Pintz's $c_{1}=2+o(1)$ result in obtaining our Theorem 2 . This gives hope to using the Burgess bounds (asymptotically giving $c_{1}=4+o(1)$ ) to improve further on our results. Explicit versions of the Burgess bounds are available (for example, see [13]). One could splice these results with the trivial and Pólya-Vinogradov estimates.

\section{References}

[1] M.A. Bennett, G. Martin, K. O’Bryant, and A. Rechnitzer. Explicit bounds for primes in arithmetic progressions. To appear in Illinois J. Math. Preprint available at arXiv: 1802.00085, 2018. 
[2] H. Davenport. Multiplicative Number Theory. Second edition. Graduate Texts in Mathematics, 74. Springer-Verlag, New York-Berlin, 1980.

[3] D.A. Frolenkov and K. Soundararajan. A generalization of the Pólya-Vinogradov inequality. Ramanujan J. 31(3), 271-279 (2013).

[4] H. Kadiri. An explicit zero-free region for Dirichlet $L$-functions. Preprint.

[5] H. Kadiri. Explicit zero-free regions for Dirichlet L-functions. Mathematika 64(2), 445474 (2018).

[6] K.S. McCurley. Explicit zero-free regions for Dirichlet L-functions. J. Number Theory 19, 7-32 (1984).

[7] M. J. Mossinghoff and T. S. Trudgian. Nonnegative trigonometric polynomials and a zero-free region for the Riemann zeta-function. J. Number Theory, 157, 329-349 (2015).

[8] M.R. Murty. Problems in Analytic Number Theory. Second edition. Graduate Texts in Mathematics, 206. Reachings in Mathematics. Springer, New York, 2008.

[9] A. Page. On the number of primes in an arithmetic progression. Proc. London Math. Soc. 39, 116-141 (1935).

[10] J. Pintz. Elementary methods in the theory of $L$-functions, V. The Theorems of Landau and Page. Acta Arith., 32, 163-171 (1977).

[11] D. Platt. Numerical computations concerning the GRH. Math. Comp. 85, 3009-3027 (2015).

[12] C. Pomerance . Remarks on the Pólya-Vinogradov inequality. Integers 11A, A19, 11pp. (2009).

[13] E. Treviño. The Burgess inequality and the least $k$-th power non-residue. Int. J. Number Theory, 11(5), 1653-1678 (2015).

[14] M. Watkins. Real zeros of real odd Dirichlet L-functions. Math. Comp. 73, 415-423 (2004). 